\title{
Combination of the multipotent mesenchymal stromal cell transplantation with administration of temozolomide increases survival of rats with experimental glioblastoma
}

\author{
IGOR BRYUKHOVETSKIY ${ }^{1,2}$, ANDREI BRYUKHOVETSKY ${ }^{3}$, YURI KHOTIMCHENKO ${ }^{1,2}$, \\ POLINA MISCHENKO ${ }^{1,2}$, ELENA TOLOK ${ }^{1,2}$ and RODION KHOTIMCHENKO ${ }^{2}$ \\ ${ }^{1}$ Laboratory of Molecular and Cellular Neurobiology, School of Biomedicine, Far Eastern Federal University, \\ Vladivostok 690091; ${ }^{2}$ Laboratory of Pharmacology, A.V. Zhirmunski Institute of Marine Biology \\ Far Eastern Branch Russian Academy of Science, Vladivostok 690041; ${ }^{3}$ NeuroVita Clinic of \\ Restorative Interventional Therapy and Neurology, Moscow 115478, Russian Federation
}

Received July 11, 2014; Accepted February 26, 2015

DOI: $10.3892 / \mathrm{mmr} .2015 .3754$

\begin{abstract}
Glioblastoma multiforme (GM) is an aggressive malignant tumor of the brain. The standard treatment of GM is surgical resection with consequent radio- and chemotherapy with temozolomide. The prognosis is unfavorable, with a survival time of 12-14 months. The phenomenon of targeted migration to the tumor in the brain opens novel possibilities for the treatment of GM. Multipotent mesenchymal stromal cells (MMSCs) are a cell type with anti-carcinogenic properties and can be used to optimize GM therapy. The aim of the present study was to investigate the effects of MMSC transplantation in the chemotherapy of a rat model of C6 glioma. A total of 130 animals were divided into a control group, a temozolomide group, MMSCs group and temozolomide + MMSCs group. The experiment was performed over 70 days, and a combination of molecular biology, surgical and neuroimaging techniques, as well as histological and physiological examinations was used. Tumor size was smallest in the temozolomide $\left(115.76 \pm 16.25 \mathrm{~mm}^{3}\right)$ and in temozolomide + MMSCs $\left(114.74 \pm 5.54 \mathrm{~mm}^{3}\right)$ groups, which was significantly smaller than the neoplastic node size in the control group $\left(202.09 \pm 39.72 \mathrm{~mm}^{3}\right)(\mathrm{P}<0.05)$. The animals in the temozolomide + MMSCs group showed significantly higher survival rates in comparison with those in the control and temozolomide groups. The MMSCs migrated from the site of implantation to the neoplastic focus and interacted
\end{abstract}

Correspondence to: Dr Igor Bryukhovetskiy, Laboratory of Molecular and Cellular Neurobiology, School of Biomedicine, Far Eastern Federal University, 8 Sukhanova Street, Vladivostok 690091, Russian Federation

E-mail: igbryukhovetsky@gmail.com

Key words: glioblastoma multiforme, temozolomide, stem cells, neural stem cells, cancer stem cells, multipotent mesenchymal stromal cells with glioma cells; however, the mechanism requires further research. In conclusion, MMSC transplantation combined with temozolomide treatment significantly extended the survival of experimental animals in comparison with those treated with temozolomide only.

\section{Introduction}

Glioblastoma multiforme (GM) is one of the most aggressive and malignant tumors of the human brain. The treatment standard of GM is surgical resection with consequent chemotherapy (1). The survival rate is 12-14 months, and cases of five-year survival are singular (2). The absence of clear borders between tumor tissue and brain substance, marked infiltration of neoplastic cells into brain parenchyma and location close to vitally important brain centers make radical removal of GM impossible (3). The tumor resistance to radio- and chemotherapy is associated with the cancer stem cells (CSCs) (4). The ability to restore damaged DNA, production of ATP-binding cassette transporters, hypoxic metabolism and the opportunity to actively interact with endothelial cells that create a barrier for cytotoxic substances make CSCs almost completely resistant to chemotherapy $(5,6)$.

To date, no efficient drugs which are able to eliminate GM CSCs have been developed; there is only the opportunity to impair separate CSC targets. For example, imatinib inhibits platelet-derived growth factor receptor and stem cell factor receptor/c-Kit signaling and blocks the mitogen-activated protein kinase signaling pathway, thus interfering with GM CSC migration (7). Rapamicin deactivates phosphatase and tensin homolog (8). Cyclopamine inhibits the Wnt/Sonic hedgehog signaling pathway in GM CSCs (9). However, these pharmaceuticals are unable to eliminate inter-phase neoplastic cells disseminated through the brain substance. To date, attempts to employ procarbazin (natulan), nimustine hydrochoride (ACNU), fotemustine, dacarbazine and irinotecan (camptosar) for this purpose have not been successful (10).

The use of monoclonal antibodies has not been efficient, either; for instance, treatment of patients of GM with antibodies to CD44 and CD133 proteins has not had any curative 
effects (11). Ipilimumab, an antibody against cytotoxic T-lymphocyte-associated protein 4 , was not able to penetrate into hypoxic areas of the tumor (12). Bevacizumab (avastin) suppresses hematopoiesis in bone marrow and increases the risk of bleeding (13). Only the tyrosine kinase inhibitors erlotinib and imatinib have produced good outcomes. However, targeted therapies have not significantly improved the survival rates of patients with GM, which may be explained by the absence of universal targets in CSCs. All targets are dynamic and are associated with specific processes that result from the functional state of the organism.

An important milestone in the development of novel approaches in GM therapy was the discovery of targeted migration of stem cells (SCs) to the neoplasm. A key factor in the process is stromal cell-derived factor-1 (SDF-1 $\alpha$ or CXCL12), a chemokine of the CXC family encoded by the CXCL12 gene (14). SDF-1 binds with CXCR4 and CXCR7 receptors of the $\mathrm{SC}$ membrane and induces migration. The process is moderated by the stem cell factor (SCF), hepatocyte growth factor, vascular endothelial growth factor, monocyte chemoattractant protein-1, high-mobility group protein B1, urokinase-type plasminogen activator and other ligands. The SCs can overcome the blood-brain barrier, affect cancer cells that are disseminated in the brain parenchyma and penetrate into hypoxic areas of GM that contain CSCs (15). However, the action of SCs on CSCs demands a specific approach, depending on their role and complexity.

The CSCs of GM are the product of the evolution of a neural stem cell (NSC) of the human brain. Common immunophenotypical clusters of differentiation, integrity of the main genes and epigenetic mechanisms that regulate key cellular processes and similarity in proteome and complete transcriptome profiles $(6,16)$ have confirmed this. NSCs are tools of local homeostasis and constantly migrate from germinal zones of the adult brain to interact with neurons and glial cells. Cellular interaction is the main mechanism of regulation of gene expression; it is an important factor of coordinated regulation of metabolism and triggers the programs of determination, differentiation, adaptation, survival, proliferation and apoptosis. Inductive interaction of the NSCs and pathologically modified cells retards their growth and initiates natural mechanisms of cell death (17).

Appropriately modified SCs can be used in conventional therapeutic protocols of GM treatment. Development of systems of somatic and stem cells with specific properties is one of the top-priority goals of biomedicine globally. At the same time, it is obvious that it will take a considerate amount of time and development until the technologies of cell reprogramming, which are based on the transfer of the somatic cell nucleus into oocyte cytoplasm, fusion of two pluripotent somatic cells and viral transfection along with other gene engineering methods, will be used in practical medicine. In spite of their advantages, possible genetic consequences and health risks of using such cell systems rule them out as the strategy of choice in GM therapy. The use of embryonic SCs in the clinic seems unlikely due to several insoluble problems: Their control in the body of a patient and serious ethical considerations as to their sources.

In a previous study by our group from 2013, comparative proteome mapping and bioinformatics analysis was performed of the lysates of neural (CD133+) stem cells isolated from the olfactory sheath of a human, multipotent mesenchymal stromal cells (CD29+, CD44+, CD73+, CD90+, CD34-) (MMSCs) isolated from the human bone marrow and CD133+ CSCs of the U87 human glioblastoma line (18). The study provided evidence that MMSCs exhibited the largest difference in their proteome profile. These cells and their progeny continuously interacted with SCs of other organs, thus completing and modulating their regulatory functions. It was therefore presumed that transplantation of this type of the cell may be a tool for enhancing the efficiency of standard protocols of GM treatment.

The goal of the present study was to assess the possible enhancing effects of MMSC transplantation in the chemotherapy of a rat model of glioblastoma.

\section{Materials and methods}

Design. A total of 130 adult Wistar rats (2-5 months-old) weighing 200-220 $\mathrm{g}$ at baseline were divided in four groups. The rats were maintained in cages at room temperature under a normal diurnal cycle, with free access to food and water. The control (first) group included C6 glioma models $(n=30)$; the second group consisted of C6 glioma rat models that received standard therapy with temozolomide $(n=30)$; the third group were C6 glioma rats that received MMSC transplantation $(n=30)$, and the fourth group consisted of C6 glioma rats received temozolomide therapy combined with MMSCs transplantation. A separate group consisted of sham-operated rats $(\mathrm{n}=10)$.

A combination of surgical, cell biological, histological and neuroimaging methods was used in the present study. The animal experiment was performed for 70 days, with particular attention given to the survival of the animals. Whenever the state deteriorated, the rats were sacrificed by deep narcosis [10 mg/kg intraperitonal injection of $200 \mu \mathrm{l}$ Zoletil 100 (Virbac, Prague, Czech Republic) + Rometar (Bioveta, Ivanovice na Hane, Czech Republic) in a 1:4 ratio]. The experiment was repeated three times. Animal care followed good laboratory practice standards and the Helsinki Declaration on the humane attitude to animals. All protocols of the present study were approved by the Ethics Committee of the School of Biomedicine of the Far Eastern Federal University (Vladivostok, Russia) (protocol no. 12 dated December 14, 2013).

Culturing of the C6 glioma cell line. The rat C6 glioma line was provided by the Laboratory of Fundamental and Applied Neurobiology of the Serbsky State Research Center of Social and Forensic Psychiatry (Moscow, Russia). The rapidly growing cell line was generated in Wistar-Furth rats by carcinogenesis induction using $N$-nitroso- $N$-methylurea; morphology, character of invasive growth and protein spectrum of C6 glioma is closest to that of human GM (19).

An aliquot of $1 \times 10^{6}$ cancer cells was defrosted for 10 minutes at $37^{\circ} \mathrm{C}$, washed with Dulbecco's modified Eagle's medium (DMEM; Gibco-BRL, Invitrogen Life Technologies, Carlsbad, CA, USA) that contained $10 \%$ fetal bovine serum (FBS; no. 26140-079; Gibco-BRL) and antibiotic-antimycotic: $10,000 \mathrm{units} / \mathrm{ml}$ penicillin, $10,000 \mu \mathrm{g} / \mathrm{ml}$ streptomycin and $25 \mu \mathrm{g} / \mathrm{ml}$ fungizone (no. 15240-062; Gibco-BRL). The cells were centrifuged $\left(120 \mathrm{x} \mathrm{g}\right.$ for $4 \mathrm{~min}$ at $\left.10^{\circ} \mathrm{C}\right)$, fresh medium 
was added, and cells were seeded into 50-ml culture flasks (Corning-Costar, Costar, NY, USA) and cultured until a monolayer developed. To split the cells, they were detached by enzyme dissociation [0.05\% trypsin-EDTA (MP Biomedicals, Santa Ana, CA, USA) 1:4; $\left.10 \mathrm{~min}, 37^{\circ} \mathrm{C}\right)$, centrifuged (120 x g, $6 \mathrm{~min}$ ), the supernatant was removed, fresh medium was added and the cells were re-suspended.

The tumor was modeled under general anesthesia $(200 \mu \mathrm{l}$ Zoletil/Rometar 1:4, intraperitoneally). The glioma cells $\left(10^{6}\right.$ cells in $20 \mathrm{ml}$ ) were implanted into the caudoputamen using a stereotaxic apparatus (Narishige, Tokyo, Japan) according to the stereotaxic coordinates of the Swanson rat atlas: Ap-1; L 3.0; V 4.5, TBS-2.4 mm (20). The cells were injected with a Hamilton syringe at a speed of $5 \mu 1 / \mathrm{min}$. Prior to injection, part of the C6 glioma cells was processed using a Vybrant ${ }^{\mathrm{B}}$ carboxyfluorescein diacetate succinimidyl ester (CFDASE) Cell Tracer (V12883; Life Technologies, Carlsbad, CA, USA) according to manufacturer's instructions.

Multipotent mesenchymal stromal cells (MMSCs). Impersonalized samples of bone marrow were provided by the ZAO Neurovita Clinic of Restorative and Interventional Neurology and Therapy (Moscow, Russia). The MMSCs were isolated using a standard procedure (21). The bone marrow sample was resuspended in RPMI-1640 medium (Sigma-Aldrich, St Louis, MO, USA) that contained 10\% FBS and $1 \%$ penicillin-streptomycin. The cells were cultured in T150 flasks (TPP, Trasadingen, Switzerland), at $37^{\circ} \mathrm{C}$ in a $5 \% \mathrm{CO}_{2}$ atmosphere. Following four days, the medium with non-adherent cells was replaced. The adhered cells were cultured to $80 \%$ confluence and passaged at 1:3. The MMSCs were characterized by surface expression of antigens (CD29+, CD44+, CD73+, CD90+, CD34-), in accordance with the manufacturer's instructions, using flow cytometry (MACSQuant ${ }^{\circledR}$ VYB; Miltenyi Biotec, Cambridge, MA, USA).

Ten days following implantation of glioma cells, $10^{6}$ MMSCs $(50 \mu \mathrm{l})$ were injected in tumor vicinity. A preliminary experiment showed that during 10 days, the size of the glioma doubled, leading to a glioma cell/MMSC ratio of 2:1. Prior to injection, all of the cells were marked with CellTracker ${ }^{\mathrm{TM}}$ Red CMTPX (C34552; Life Technologies) according to the manufacturer's instructions. Sham-operated rats received injections of $20 \mu$ l DMEM.

Chemotherapy. The standard of GM chemotherapy is the treatment with temozolomide, a cytostatic anti-tumor alkalyting agent. The agent rapidly reaches the systemic blood supply and is processed into an active metabolite whose cytotoxic action is determined by its ability to disturb the structure and synthesis of DNA, which most often occurs at the N-7 or O-6 positions of guanine residues (22). The present study used temozolomide under the brand name temodal (MSD Shering-Plough Labo, Heist-op-den-Berg, Belgium). Magnetic resonance imaging (MRI) was performed on the rats ten days after glioma cell implantation. If the tumor was clearly imaged, the animals of the appropriate groups received $50 \mu \mathrm{g} / \mathrm{kg}$ temozolomide orally from days 10-14 of the experiment.

Neuroimaging. MRI of the brain was performed using a Biospec MR tomograph (Bruker, Billerica, MA, USA) with a special magnetic coil for small laboratory animals $(2-3 \mathrm{~cm}$ inner diameter) under general anesthesia. MRI was performed at days 10, 20, 30, 40, 50, 60 and 70 of the trial.

Neurological status and body weight. Examination of the neurological status in rats was accomplished according to the standard algorithm (23). The body weight was measured using a Sartorius CPA12001S (Sartorius, Göttingen, Germany) laboratory balance.

Histological study. The 40- $\mu \mathrm{m}$ thick sections were stained with cresyl violet, toluidine blue, hematoxylin and eosin (all Sigma-Aldrich) according to standard protocols, and with vanadium acid fuchsine (Sigma-Aldrich), according to the Victorov method (24). The brain sections were studied using a Leica DM 6000 (Leica Microsystems GmbH, Wetzlar, Germany).

Tumor morphometry. The size of the tumor was defined according to the formula: $\mathrm{V}=4 \backslash 3 \pi \mathrm{abc}$, where $\mathrm{a}, \mathrm{b}$ and $\mathrm{c}$ are semi-axes of an ellipsoid. Primarily, the section with the maximal glioma area was detected, where a large semi-axis (a) and a small semi-axis (b) of the ellipsoid were defined. Then, the lengths of the frontal sections from the anterior to the posterior side of a tumor node were summed, where the anterior-posterior semi-axis was then defined (c) using a Biospec MR tomograph.

Statistical analysis. Statistical analysis was performed using Excel 2010 (Microsoft Corp., Redmond, WA, USA). Image processing and graphical analysis was performed with ImageJ 1.43 software (National Institutes of Health, Bethesda, MD, USA).

\section{Results}

Tumor morphology. Stereotaxic implantation of C6 glioma cells to the rat brain led to tumor formation (Fig. 1A). The T2-Turbo RARE mode scans showed voluminous neoplasms of irregular shape with signs of compression of brain ventricles and other structures, as well as foci of hemorrhages and edema in the brain structure (Fig. 1B). Histological analysis demonstrated an extensive neoplasm with unclear borders and edema of the neighboring white matter. The tumor consisted of cells of different shapes and sizes with different numbers of nuclei. The tumor spread into the perivasal and perineural spaces. Acidophilic cells were selectively stained red with vanadium acid fuchsine and extensive areas of central necrosis that occasionally transformed in cysts were found. Fluorescence microscopy $(\lambda=488 \mathrm{~nm})$ showed a heterogeneous distribution of neoplastic elements in the tumor parenchyma and the tendency to migrate to the neighboring tissues (Fig. 1C-H).

MMSCs increase the survival of a rat model of GM treated with temozolomide. The average survival of the control group animals was $27.2 \pm 5.2$ days from the moment of surgery (Fig. 2). The animals lost body weight rapidly (Fig. 3), were inert, disinterested in the events in the cage, refused to feed and were reluctant to drink. Attempts to pick up an animal by hand or slight contact with the vibrissae caused reactions 

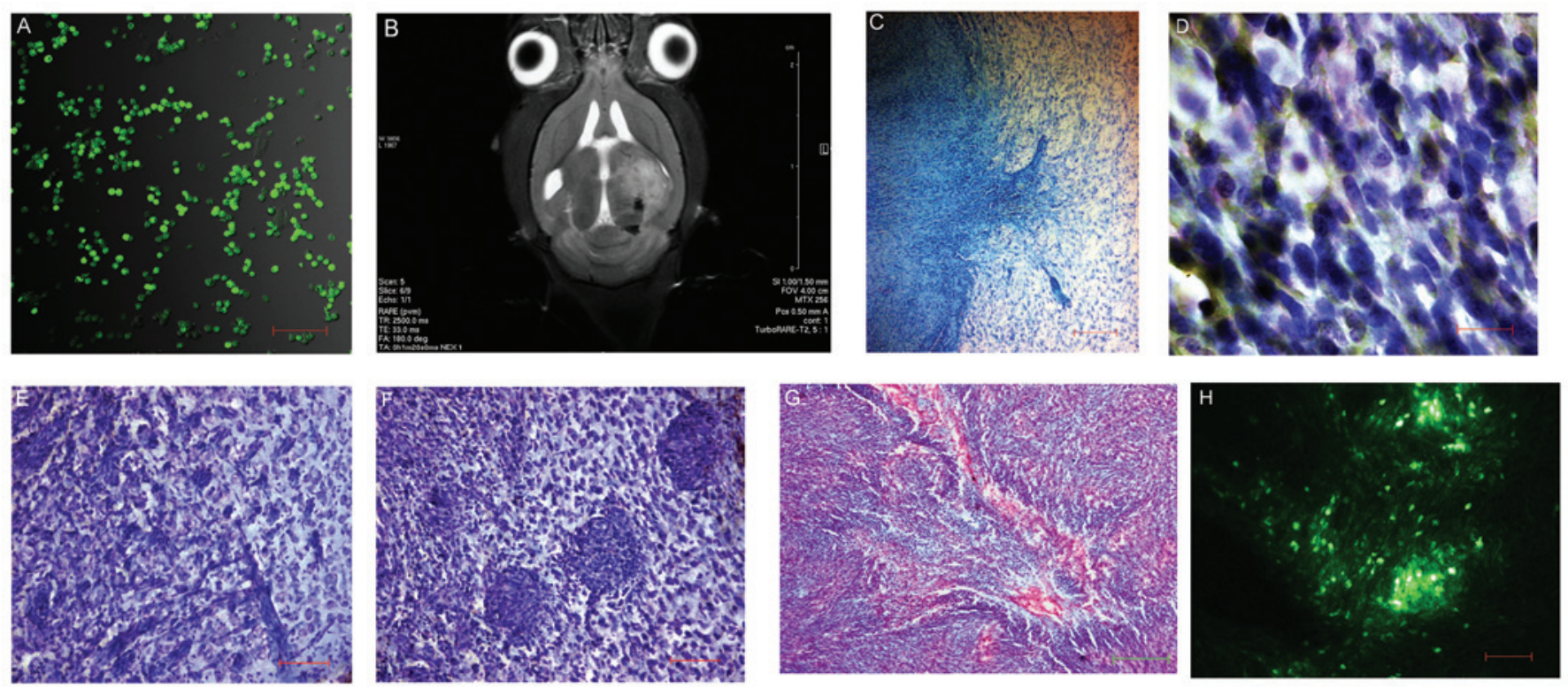

Figure 1. (A) Vybrant ${ }^{\circledR}$ CFDASE fluorescence of C6 glioma cells in the culture prior to implantation. LSM T-PMT Carl Zeiss Aim-system, (laser, $\lambda=488 \mathrm{~nm}$; scale bar, $200 \mu \mathrm{m}$ ). (B) Magnetic resonance thermogram of the rat brain seven days post C6 glioma cell transplantation. TurboRARE-T2 mode. Ventricle compression, edema and disposition of median brain structures are observed. (C) Area of invasive growth of C6 glioma. Toluidine blue staining (scale bar, $100 \mu \mathrm{m}$ ). The tumor borders are unclear; invasion area and infiltration of neoplastic elements into the brain substance are observed. (D) Polymorphism of C6 glioma cell nuclei. Cresyl violet and toluidine blue staining (oil immersion; scale bar, $10 \mu \mathrm{m}$ ). (E) Perineural invasion of C6 glioma. Cresyl violet and toluidine blue staining (scale bar, $100 \mu \mathrm{m}$ ). (F) Perivasal invasion of C6 glioma. Cresyl violet and toluidine blue staining (scale bar, $100 \mu \mathrm{m}$ ). (G) Central area of necrosis of C6 glioma. Staining according to the method of I. Viktorov. Acidophilic cells are selectively stained red (scale bar, $200 \mu \mathrm{m}$ ). (H) C6 glioma node in the rat brain. Cells were stained using Vybrant ${ }^{\circledR}$ CFDASE Cell Tracer. LSM T-PMT Carl Zeiss Aim-system 2601876 (laser, $\lambda=488 \mathrm{~nm}$; scale bar, $100 \mu \mathrm{m}$ ). LSM, laser scanning microscope; CFDASE, carboxyfluorescein diacetate succinimidyl ester.

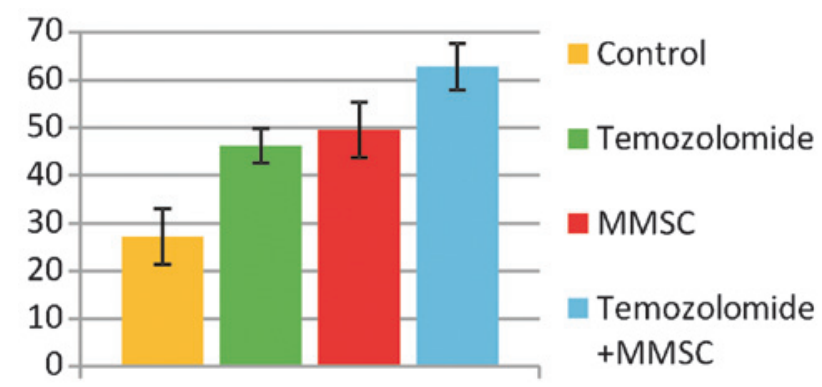

Figure 2. Survival (days) of the experimental animals. P<0.05, MMSC group vs. Control group, Temozolomide + MMSC group vs. Control and Temozolomide groups. MMSC, multipotent mesenchymal stromal cells.

of loud squeaking. Mild neurological symptoms, including ptosis, tremor and paresis of extremities, rapidly aggravated, resulting in paralysis with exophthalmos followed by coma and respiratory impairments that appeared to be a result of brain dislocation. The sham-operated rats showed no significant changes in body weight and functional status, and survived until the end of the experiment with no complications.

The average survival time of the temozolomide-treated animals was $46.2 \pm 3.6$ days from the start of the experiment (Fig. 2). The animals in this group showed no significant differences in body weight in comparison with that of the controls (Fig. 3); furthermore, they were reluctant to eat and were inert. The general condition of the animals was less severe than that of the control animals; it was more stable and the development of acute neurological disorders was less abrupt. Brain symptoms manifested as bilateral hemoptosis and mild spastic paresis of extremities in combination with

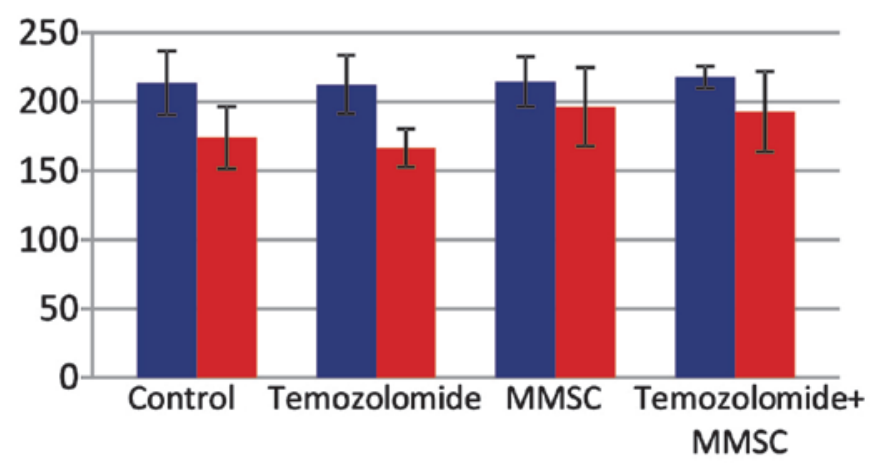

-At the beginning of the study $\square$ At the end of the experiment

Figure 3. Changes in body weight $(\mathrm{g})$ of the experimental animals. MMSC, multipotent mesenchymal stromal cells.

spasms of the paws. Inhibition was replaced by the episodes of excitation that manifested in circular movements within the cage associated with the damage of one of the hemispheres. Morphological changes in the brains of the animals excluded from the experiment due to illness showed no differences from the morphological changes in the control group.

The average survival time of the animals with C6 glioma tumors that received transplantation of MMSCs was $49.56 \pm 1.94$ days from the start of the experiment, which was significantly different from that of the controls, but not different from that in the temozolomide-treated group. Neurological examination showed minor neurological symptoms: Reduced corneal reflex, ptosis and exophthalmos on the tumor side, 

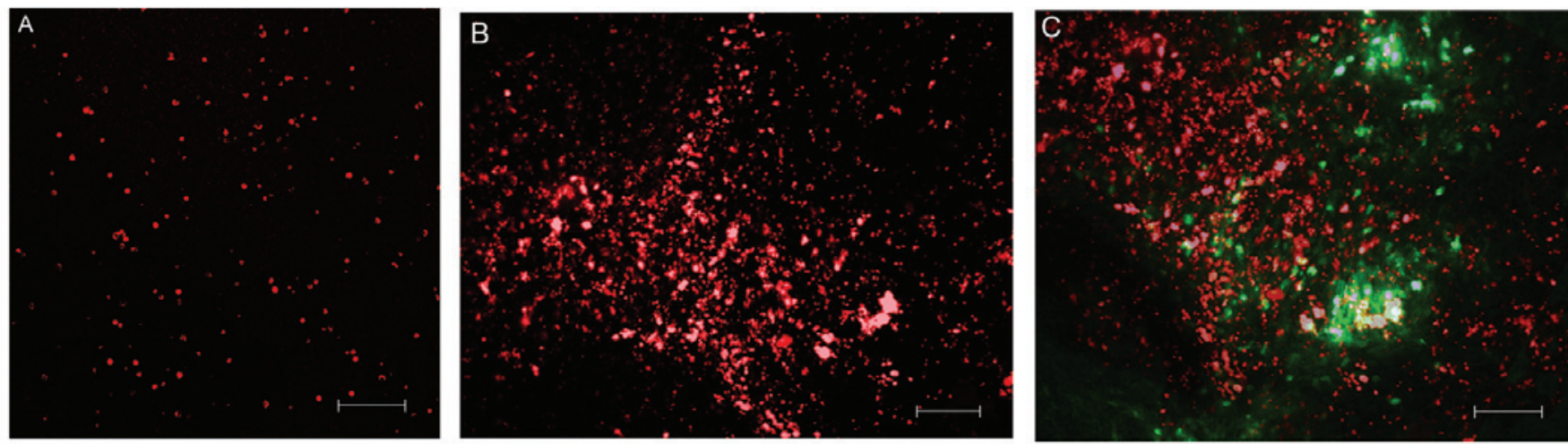

Figure 4. (A) CMTPX-positive MMSCs (red) prior to transplantation. LSM T-PMT Carl Zeiss Aim-system 2601876 (laser, $\lambda=650 \mathrm{~nm}$; scale bar, $100 \mu \mathrm{m}$ ). (B) Migration of CMTPX-positive MMSCs (red) at day 10 in the brain substance adjacent to the neoplasm (scale bar, $100 \mu \mathrm{m}$ ). (C) CMTPX-positive MMSCs (laser fluorescence, $\lambda=650 \mathrm{~nm}$, red) in the tumor parenchyma and the tissue adjacent to the tumor nodes formed by CFDASE-positive tumor cells (laser fluorescence, $\lambda=488 \mathrm{~nm}$, green). Overlay of red and green fluorescence (scale bar, $100 \mu \mathrm{m}$ ). MMSCs, multipotent mesenchymal stromal cells; LSM, laser scanning microscopy; CFDASE, carboxyfluorescein diacetate succinimidyl ester.

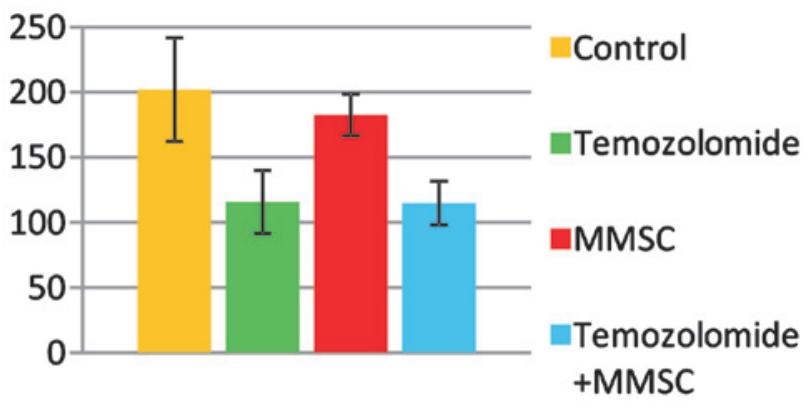

Figure 5. Size of gliomas in experimental animals, $\mathrm{mm}^{3}$. $\mathrm{P}<0.05$, Control and MMSC groups vs. Temozolozide and Temozolozide + MMSC groups. MMSC, multipotent mesenchymal stromal cells.

reduced flexor and grasp reflexes, increased reaction to light and sound stimuli, and retardation in the head shaking test (22). The animals remained more active for a longer time as compared with the controls, did not refuse to eat and did not lose weight significantly. Histological analysis showed an extensive tumor formation with unclear borders and areas of invasion to the brain substance (Fig. 1C).

MMSCs were observed at the site of injection and at a certain distance from it. The cells were ball-shaped and no branching was present. It appeared that during glioma growth, the MMSCs migrated following neoplastic cells and possibly interacted with them. Overlaying of red and green fluorescence showed that the MMSCs had accumulated along the tumor borders and were also present in the parenchyma of the neoplasm (Fig. 4A-C). MMSCs were not visible by fluorescence microscopy at day 30 of the trials, which may have been due to their death, differentiation or involvement in the tumor biological process.

The survival time of the animals that received chemotherapy along with MMSC transplantation was $62.8 \pm 4.85$ days, which is significantly longer than the survival times in the control and temozolomide groups. The animals showed no significant weight loss, were active and did not refuse to eat. Neurological examination showed mild neurological symptoms with further abrupt coma and decreased viability.

The tumor size varied considerably among the groups (Fig. 5). The smallest size was found in the temozolomide-treated group $\left(115.76 \pm 16.25 \mathrm{~mm}^{3}\right)$ and in the temozolomide + MMSCs group $\left(114.74 \pm 5.54 \mathrm{~mm}^{3}\right)$, which were significantly smaller than the size of neoplastic nodes in the control group $\left(202.09 \pm 39.72 \mathrm{~mm}^{3}\right)$ and in the MMSCs group $\left(182.72 \pm 15.96 \mathrm{~mm}^{3}\right)(\mathrm{P}<0.05)$.

\section{Discussion}

From an evolutionary perspective and with regard to the antagonism between CSCs and healthy NSCs, tumor development can be understood as the result of local functional dominance of the neoplastic SCs. Among the CSCs of the C6 glioma cel line, $87.24 \%$ are CD133+ (25), making it advantageous for the use in models of GM as compared with other glioma cell lines (26). Transplantation of C6 glioma cells leads to the collapse of autoregulation mechanisms of tissue homeostasis in the rat brain, and the quantitative advantage of CD133+ CSCs permits the C6 cell line to rapidly develop vascular, lymphatic and neural networks, to optimize metabolism and to trigger invasive processes at a high speed, which explains the severity of GM and the high mortality rates in the control group of the present study.

Temozolomide significantly reduces the size of glioma, which was proven by the present and previous studies (27). The cytotoxic activity of the agent reduces the size of the tumor nodes, but considerably enhances hypoxia $(28,29)$, which appears to promote the production of chemokines that induce processes of targeted migration of SCs to the tumor focus. Factors including healthy SCs that migrated to the tumor abruptly shifted this balance, explaining for the increased survival as the main indicator of partial restoration of the system.

The transplanted MMSCs were able to slow down tumor cell proliferation, which additionally made the latter susceptible to the regulatory signals of apoptosis and autophagy (30). Activation of tumor necrosis factor (TNF), TNF-related apoptosis-inducing ligand, nerve-, insulin-like and endothelial growth factor receptors, CAR1, CD95 receptors and DR3, DR4, DR5 trans-membrane proteins in the SCs induces apoptosis in tumor cells. In co-culture with MMSCs, the cyclins E and D2 as well as p27KIPl accumulate in the medium, thus blocking proliferation of tumor cells in G0/G1 phase (31). Death of 
C6 glioma cells upon interaction with SCs can be caused by disorders in the intracellular homeostasis of calcium ions, and a specific signal is transferred through cell junctions by means of bone morphogenetic protein 4 and interleukin-1 (32). Neuroplastic action of SCs and the production of biologically active substances by SCs in the pathological tissue also can be regarded as mechanisms of their anti-cancer action (33).

It should be noted that the fate of xenotransplantation in the present study was initially defined. A certain degree of immune suppression caused by hormones or cytostatics can delay the death of the transplanted cell systems. Thus, in the context of the present study, MMSCs can be viewed as cell systems which induced apoptosis. A previous study by our group reported the possibility of apoptosis induction in cells of glial tumors during interaction with NSCs and hematopoiesis precursors. When these cell systems were co-cultured with C6 glioma and U87 glioblastoma cells, they induced apoptosis in them and slowed down the neoplastic process (34). It is possible that this mechanism reduced the size of tumor nodes and ameliorated the general condition of the rats.

However, GM is able to recruit normal somatic cells and SCs. The tumor uses their potential and involves them in carcinogenesis. Liu et al (35) reported that SCs that had been co-cultured with tumor cells underwent malignant transformations. It cannot be ruled out that the death of tumor cells activates survival mechanisms in CSCs and functions as a factor that selects the most aggressive and resistant cells in the tumor microenvironment.

The results of the present study lead to an important conclusion: The rats with C6 glioma that received chemotherapy in combination with MMSCs survived significantly longer than the animals that received temozolomide only $(\mathrm{P}<0.05)$. Therefore, the survival, which is the main criterion of therapeutic efficiency in oncology, significantly improved when stem cell transplantation was used. Identification of the mechanisms of this phenomenon and development of more sensitive and accurate methods to control cell induction is among priority tasks of studies to be performed in the near future. Due to the evidence provided, the MMSC preparations can be viewed as promising tools of regulation and management of neoplastic processes in the glial tumor and open opportunities for the development of novel therapeutic strategies against neurooncological diseases.

\section{Acknowledgements}

The present study was funded by the Russian Science Fund (project no. 14-15-00084).

\section{References}

1. Louis DN, Ohgaki H, Wiestler OD, Cavenee WK, Burger PC, Jouvet A, Scheithauer BW and Kleihues P: The 2007 WHO classification of tumours of the central nervous system. Acta Neuropathol 114: 97-109, 2007.

2. Hottinger AF, Stupp R and Homicsko K: Standards of care and novel approaches in the management of glioblastoma multiforme. Chin J Cancer 33: 32-39, 2014.

3. Goffart N, Kroonen J and Rogister B: Glioblastoma-initiating cells: Relationship with neural stem cells and the micro-environment. Cancers (Basel) 5: 1049-1071, 2013.
4. Pointer KB, Clark PA, Zorniak M, Alrfaei BM and Kuo JS: Glioblastoma cancer stem cells: Biomarker and therapeutic advances. Neurochem Int 71: 1-7, 2014.

5. Ramirez YP, Weatherbee JL, Wheelhouse RT and Ross AH: Glioblastoma Multiforme therapy and mechanisms of resistance. Pharmaceuticals (Basel) 6: 1475-1506, 2013.

6. Bryukhovetskyi IS, Bryukhovetskyi AS, Kumeiko VV, Mishenko PV and Khotimchenko YS: Stem cells in carcinogenesis of glioblastoma multiforme. Genes Cells 8: 13-19, 2013 (In Russian).

7. Dong Y, Han Q, Zou Y, Deng Z, Lu X, Wang X, Zhang W, Jin H, $\mathrm{Su} \mathrm{J}$, Jiang T, et al: Long-term exposure to imatinib reduced cancer stem cell ability through induction of cell differentiation via activation of MAPK signaling in glioblastoma cells. Mol Cell Biochem 370: 89-102, 2012.

8. Mendiburu-Eliçabe M, Gil-Ranedo J and Izquierdo M: Efficacy of rapamycin against glioblastoma cancer stem cells. Clin Transl Oncol 16: 495-502, 2014.

9. Eimer S,Dugay F,Airiau K, AvrilT, Quillien V,Belaud-Rotureau MA and Belloc F: Cyclopamine cooperates with EGFR inhibition to deplete stem-like cancer cells in glioblastoma-derived spheroid cultures. Neuro-oncol 14: 1441-1451, 2012.

10. Parney IF and Chang SM: Current chemotherapy for glioblastoma. Cancer J 9: 149-156, 2003.

11. Tamura K, Aoyagi M, Ando N, Ogishima T, Wakimoto H, Yamamoto M and Ohno K: Expansion of CD133-positive glioma cells in recurrent de novo glioblastomas after radiotherapy and chemotherapy. J Neurosurg 119: 1145-1155, 2013.

12. Brower V: Early-stage progress on glioma vaccines. J Natl Cancer Inst 103: 1361-1362, 2011.

13. Rovere RK: Bevacizumab as secondline treatment of glioblastoma - worth the effort? Klin Onkol 27: 219-220, 2014.

14. Nagasawa T: CXC chemokine ligand 12 (CXCL12) and its receptor CXCR4. J Mol Med (Berl) 92: 433-439, 2014.

15. Bryukhovetskyi IS, Bryukhovetskyi AS, Mischenco PV and Khotimchenko YS: The role of systemic migration and homing mechanisms of stem cells in the development of malignant tumors of the central nervous system and the development of new cancer therapies. Russian Biotherapeutic J 4: 3-12, 2013 (In Russian).

16. Rispoli R, Conti C, Celli P, Caroli E and Carletti S: Neural stem cells and glioblastoma. Neuroradiol J 27: 169-174, 2014.

17. Paltsev MA, Ivanov AA and Severin SE: [Intercellular interactions]. Medicine (Moscow), 2003. (In Russian)

18. Bryukhovetskiy AS, Shevchenko VE, Chekhonin VP, Bryukhovetskiy IS, Kovalev SV, Baklaushev VP and Davydov MI: Comparative proteome mapping of tumor stem cells isolated from U87 glioblastoma, neural stem and multipotent mesenchymal stromal cells of human: From cataloguing of cell proteins to novel paradigm of proteome-based cell therapy of tumors. Genes Cells 8: 85-92, 2013.

19. Grobben B, De Deyn PP and Slegers H: Rat C6 glioma as experimental model system for the study of glioblastoma growth and invasion. Cell Tissue Res 310: 257-270, 2002.

20. Swanson LW: Brain Maps: Structure of the Rat Brain. 2nd edition. Elsevier, Amsterdam, 1998.

21. Pittenger MF: Mesenchymal stem cells from adult bone marrow. Methods Mol Biol 449: 27-44, 2008.

22. Shen W, Hu JA and Zheng JS: Mechanism of temozolomide-induced antitumour effects on glioma cells. J Int Med Res 42: 164-172, 2014.

23. Tupper DE and Wallace RB: Utility of the neurological examination in rats. Acta Neurobiol Exp (Wars) 40: 999-1003, 1980.

24. Victorov IV, Prass K and Dirnagl U: Improved selective, simple, and contrast staining of acidophilic neurons with vanadium acid fuchsin. Brain Res Brain Res Protoc 5: 135-139, 2000.

25. Shen G, Shen F, Shi Z, Liu W, Hu W, Zheng X, Wen L and Yang X: Identification of cancer stem-like cells in the C6 glioma cell line and the limitation of current identification methods. In Vitro Cell Dev Biol Anim 44: 280-289, 2008.

26. Barth RF and Kaur B: Rat brain tumor models in experimental neuro-oncology: The C6, 9L, T9, RG2, F98, BT4C, RT-2 and CNS-1 gliomas. J Neurooncol 94: 299-312, 2009.

27. Hirst TC, Vesterinen HM, Sena ES, Egan KJ, Macleod MR and Whittle IR: Systematic review and meta-analysis of temozolomide in animal models of glioma: Was clinical efficacy predicted? Br J Cancer 108: 64-71, 2013.

28. Steinbach JP, Wolburg H, Klumpp A, Probst H and Weller M: Hypoxia-induced cell death in human malignant glioma cells: Energy deprivation promotes decoupling of mitochondrial cytochrome $c$ release from caspase processing and necrotic cell death. Cell Death Differ 10: 823-832, 2003. 
29. Yang L, Lin C, Wang L, Guo H and Wang X: Hypoxia and hypocia-inducible factors in glioblastoma multiforme progression and therapeutic implications. Exp Cell Res 318: 2417-2426, 2012

30. Zhang MH, Hu YD, Xu Y, Xiao Y, Luo Y, Song AC and Zhou J: Human mesenchymal stem cells enhance autophagy of lung carcinoma cells against apoptosis during serum deprivation. Int J Oncol 42: 1390-1398, 2013.

31. Hou L, Wang X, Zhou Y, Ma H, Wang Z, He J, Hu H, Guan W and Ma Y: Inhibitory effect and mechanism of mesenchymal stem cells on liver cancer cells. Tumour Biol 35: 1239-1250, 2014.

32. Li Q, Wijesekera O, Salas SJ, Wang JY, Zhu M, Aprhys C, Chaichana KL, Chesler DA, Zhang H, Smith CL, et al: Mesenchymal stem cells from human fat engineered to secrete BMP4 are nononcogenic, suppress brain cancer, and prolong survival. Clin Cancer Res 20: 2375-2387, 2014.
33. Baraniak PR and McDevitt TC: Stem cell paracrine actions and tissue regeneration. Regen Med 5: 121-143, 2010.

34. Bryukhovetskiy A, Bryukhovetskiy I, Chekhonin V and Baklaushev V: Experimental cytoregulatory therapy of brain glial tumors with cell system of hematopoietic precursors epigenetically reprogrammed of apoptosis induction: Victory in vitro and failure in vivo. J Neurol 257 (Suppl 1): S152-S153, 2010.

35. Liu J, Zhang Y, Bai L, Cui X and Zhu J: Rat bone marrow mesenchymal stem cells undergo malignant transformation via indirect co-cultured with tumour cells. Cell Biochem Funct 30: 650-656, 2012. 